\title{
Analysis-Error Statistics of a Quasigeostrophic Model Using Three-Dimensional Variational Assimilation
}

\author{
ThOMAS M. HAMILL \\ NOAA-CIRES Climate Diagnostics Center, Boulder, Colorado \\ Chris SNyder and Rebecca E. Morss \\ National Center for Atmospheric Research, * Boulder, Colorado
}

(Manuscript received 17 December 2001, in final form 5 April 2002)

\begin{abstract}
A perfect model Monte Carlo experiment was conducted to explore the characteristics of analysis error in a quasigeostrophic model. An ensemble of cycled analyses was created, with each member of the ensemble receiving different observations and starting from different forecast states. Observations were created by adding random error (consistent with observational error statistics) to vertical profiles extracted from truth run data. Assimilation of new observations was performed every $12 \mathrm{~h}$ using a three-dimensional variational analysis scheme. Three observation densities were examined, a low-density network (one observation $\sim$ every $20^{2}$ grid points), a moderate-density network (one observation $\sim$ every $10^{2}$ grid points), and a high-density network $(\sim$ every $5^{2}$ grid points). Error characteristics were diagnosed primarily from a subset of 16 analysis times taken every 10 days from a long time series, with the first sample taken after a 50-day spinup. The goal of this paper is to understand the spatial, temporal, and some dynamical characteristics of analysis errors.

Results suggest a nonlinear relationship between observational data density and analysis error; there was a much greater reduction in error from the low- to moderate-density networks than from moderate to high density. Errors in the analysis reflected both structured errors created by the chaotic dynamics as well as random observational errors. The correction of the background toward the observations reduced the error but also randomized the prior dynamical structure of the errors, though there was a dependence of error structure on observational data density. Generally, the more observations, the more homogeneous the errors were in time and space and the less the analysis errors projected onto the leading backward Lyapunov vectors. Analyses provided more information at higher wavenumbers as data density increased. Errors were largest in the upper troposphere and smallest in the mid- to lower troposphere. Relatively small ensembles were effective in capturing a large percentage of the analysis-error variance, though more members were needed to capture a specified fraction of the variance as observation density increased.
\end{abstract}

\section{Introduction}

The purpose of this paper is to understand better some of the characteristics of analysis errors for a three-dimensional variational (3DVAR) data assimilation analysis system. We seek to understand the general characteristics of analysis errors, such as how they depend on observational density and their spatial and temporal variability. We also seek a quantitative understanding of how much the analysis error statistics reflect the dynamically structured errors contributed from the back-

* The National Center for Atmospheric Research is sponsored by the National Science Foundation.

Corresponding author address: Dr. Thomas M. Hamill, NOAACIRES Climate Diagnostics Center, R/CDC 1, 325 Broadway, Boulder, CO 80305-3328.

E-mail: hamill@cdc.noaa.gov ground state and how much they reflect the random errors from the observations and their assimilation. Companion papers Snyder et al. (2002, hereafter SNY) and Synder and Hamill (2001, manuscript submitted to J. Atmos. Sci., hereafter SNHA) will explore the dynamical mechanisms for error growth during the forecast and will demonstrate how these forecast error statistics can be strongly anisotropic, inhomogeneous, and nonstationary. Our focus here is on how these dynamically structured forecast errors are modified during the analysis process.

These issues are germane for making progress in both data assimilation and probabilistic weather prediction. For example, in data assimilation, many new ensemblebased data assimilation approaches have been proposed (e.g., Evensen 1994; Evensen and van Leeuwen 1996; Houtekamer and Mitchell 1998, 2001; Burgers et al. 1998; Mitchell and Houtekamer 2000, 2002; Lermusiaux and Robinson 1999; van Leeuwen 1999; Anderson 
and Anderson 1999; Hamill and Snyder 2000; Heemink et al. 2001; Hansen and Smith 2001; Keppenne 2000; Anderson 2001; Hamill et al. 2001; Whitaker and Hamill 2002; Pham 2001). All of these techniques assume that background-forecast errors (and by extension, the analysis errors that gave rise to them) can be accurately modeled in a severely reduced dimensional subspace. Though some research has touched on the issue (e.g., Tippett et al. 2000), the validity of low-dimensional approximations to error covariance statistics in complex models has not been rigorously explored, nor is it generally understood why low-dimensional representations may be valid.

For similar reasons, these issues are also relevant to ensemble forecasting and probabilistic prediction. Ensemble forecast methods use a sample of weather forecasts to estimate the probability of subsequent forecast states (Leith 1974). Whether initial conditions are generated from random samples or under dynamical constraints, there is general agreement that they should be sampled from the probability distribution of possible analysis states (e.g., Ehrendorfer and Tribbia 1997). Presuming the forecast model faithfully represents the atmosphere's dynamics, an ensemble of forecasts from these initial conditions should provide a reasonable sample of future forecast states. The ensemble can then be used to make probabilistic forecasts. Though the analysis-error distribution is the one that should be sampled, until recently the characteristics of this distribution had not been explored very thoroughly or estimated very well (however, note again, that new ensemble-based data assimilation methods offer the hope of sampling this distribution more accurately).

The characteristics of analysis errors may be important for determining the preferred method for generating initial conditions for ensemble forecasts. It has often been presumed that Monte Carlo methods that randomly sampled the analysis probability distribution would not be particularly useful, owing to the huge dimensionality of the state space (and presumably the huge effective dimensionality of the analysis distribution). Since large ensembles are not computationally feasible, it was presumed that smaller ensembles with random perturbations were likely to be inefficient at modeling the error evolution during the forecast (e.g., Mureau et al. 1993; Toth and Kalnay 1993; Ehrendorfer and Tribbia 1997). Hence, many have suggested that initial conditions should project upon the dynamically growing features contained within the subspace of analysis errors. Accordingly, operational medium-range ensemble forecasts in the United States and Europe have taken the approach of specially selecting initial conditions where error growth has been large in the past (e.g., Toth and Kalnay 1993, 1997) or is expected to be large in the future (e.g., Molteni et al. 1996), respectively. In fact, in the methodologies currently used in the United States and Europe, generating samples with growing error structures is considered more important than designing samples that closely match analysis-error statistics (though see Barkmeijer et al. 1998, 1999).

However, if the analysis-error distribution is itself relatively low in dimension compared with the dimension of the state space, a small ensemble randomly sampled from this distribution may be able to efficiently estimate future probabilities. Experimental comparisons of Monte Carlo and nonrandom methods for generating initial conditions suggests that appropriately designed Monte Carlo methods can be competive or even superior (e.g., Anderson 1996; Hamill et al. 2000) to approaches that consider error-growth dynamics but not analysis-error statistics. However, neither of these studies systematically documented the characteristics of analysis errors. Evidence in the companion paper (SNY) suggests that the forecast dynamics quickly organize errors so that they project significantly on a reduced-dimension subspace. Will the organized errors substantially "survive" the analysis process? Assimilating observations with random errors and reducing the errors preferentially in the growing directions may substantially whiten the errors, that is, make the analysis-error distribution higher dimensional. The extent of this effect has not been thoroughly explored.

Our approach here will be to explore the characteristics of analysis errors in an idealized system, a system where large ensembles and a long period of data assimilations are possible and where the model true state is known. Because of its simplicity and widespread use, we have chosen to use a 3DVAR analysis system (e.g., Parrish and Derber 1992, hereafter PD92; Lorenc et al. 2000; Ingleby 2001). We have also chosen to use a quasigeostrophic (QG) channel model. The QG model, of course, has balanced dynamics and in this respect is thus an imperfect analog to the dynamics of primitive equation models. However, this model has a large number of degrees of freedom, and this model has many of the salient characteristics of weather prediction models (SNY). We also acknowledge that the perfect-model assumption is a limitation of this study, since in operational weather forecasting the model errors contribute significantly to overall forecast errors and may affect the amount of randomization in the analysis. However, its use does permit us to understand more thoroughly the connections of analysis and background error with the forecast dynamics and the data assimilation methodology, uncomplicated by other error sources.

In order to generate robust statistics of the errors and to examine their instantaneous structure, we will run an ensemble of assimilation cycles. Unique observations will be assimilated by each member. These observations are generated by adding different random errors to the true observations, extracted from the true model state. This is roughly analogous to the perturbed observation approach (Houtekamer and Derome 1995; Houtekamer et al. 1996; Hamill et al. 2000), though here we have an ensemble where each member is receiving a realistic observation (noise added to the perfect observation), 
TABLE 1. Temperature observational error covariances $\left(\mathrm{K}^{2}\right)$.

\begin{tabular}{ccccccccc}
\hline \hline Level & 1 & 2 & 3 & 4 & 5 & 6 & 7 & 8 \\
\hline 1 & 2.82 & 1.97 & 1.15 & 0.72 & 0.52 & 0.42 & 0.35 & 0.29 \\
2 & 1.97 & 2.06 & 1.52 & 0.98 & 0.67 & 0.52 & 0.41 & 0.33 \\
3 & 1.15 & 1.52 & 1.69 & 1.38 & 0.97 & 0.71 & 0.53 & 0.41 \\
4 & 0.72 & 0.98 & 1.38 & 1.69 & 1.51 & 1.13 & 0.81 & 0.59 \\
5 & 0.52 & 0.67 & 0.97 & 1.51 & 2.01 & 1.92 & 1.41 & 0.98 \\
6 & 0.42 & 0.52 & 0.71 & 1.13 & 1.92 & 2.74 & 2.56 & 1.83 \\
7 & 0.35 & 0.41 & 0.53 & 0.81 & 1.41 & 2.56 & 3.59 & 3.24 \\
8 & 0.29 & 0.33 & 0.41 & 0.59 & 0.98 & 1.83 & 3.24 & 4.39 \\
\hline
\end{tabular}

not a degraded, perturbed observation (noise added to an imperfect observation). Ensembles from either approach will generate very similar covariance statistics; the advantage of the former over the latter is that we have independent realizations of realistic analyses rather than degraded analyses from the use of perturbed observations. With an ensemble time series of analyses and short-range forecasts, we can answer some interesting questions about the characteristics of analysis errors. These include questions about the magnitude of the error and how it changes with observational density, the spatial and temporal variability of errors, the error correlation structures, and attributes relevant to ensemble forecasting, such as the amount of variance explained as a function of ensemble size.

Section 2 will outline the experimental design in further detail; section 3 provides a description of the analysis system, and section 4 discusses the results. Section 5 concludes.

\section{Experimental design}

Our experiments begin with the assumption of a perfect model. Thus, a long reference integration of a quasigeostrophic (QG) channel model provided the true state; the assimilation experiments then used that same model together with imperfect simulated observations of the true state.

The quasigeostrophic model used here was used in Hamill et al. (2000) and Hamill and Snyder (2000, 2002), and is documented extensively in SNY. It is a midlatitude, beta-plane, gridpoint channel model that is periodic in $x$ (east-west), has impermeable walls on the north-south boundaries, and rigid lids at the top and bottom. There is no terrain, nor are there surface variations such as land and water. Pseudo-potential vorticity $q$ is conserved except for Ekman pumping at the surface, $\nabla^{4}$ horizontal diffusion, and forcing by relaxation to a zonal mean state. The domain is $16000 \mathrm{~km} \times 8000$ $\mathrm{km} \times 9 \mathrm{~km}$; there are 129 grid points east-west, 65 north-south, and eight model forecast levels, with additional staggered top and bottom levels at which potential temperature $\theta$ is specified, a total of 83850 degrees of freedom. Forecast parameters are set as in Hamill et al. (2000).

For these experiments, all observations were pre-
TABLE 2. Wind observational error covariances $\left(\mathrm{m}^{2} \mathrm{~s}^{-2}\right)$.

\begin{tabular}{ccccccccc}
\hline \hline Level & 1 & 2 & 3 & 4 & 5 & 6 & \multicolumn{1}{c}{7} & \multicolumn{1}{c}{8} \\
\hline 1 & 2.70 & 3.04 & 2.56 & 1.88 & 1.40 & 1.07 & 0.85 & 0.65 \\
2 & 3.04 & 4.35 & 4.61 & 3.59 & 2.63 & 1.94 & 1.48 & 1.10 \\
3 & 2.56 & 4.61 & 6.21 & 5.84 & 4.52 & 3.29 & 2.42 & 1.74 \\
4 & 1.88 & 3.59 & 5.84 & 7.27 & 6.80 & 5.23 & 3.80 & 2.63 \\
5 & 1.40 & 2.63 & 4.52 & 6.80 & 8.42 & 7.83 & 6.00 & 4.10 \\
6 & 1.07 & 1.94 & 3.29 & 5.23 & 7.83 & 9.64 & 8.93 & 6.44 \\
7 & 0.85 & 1.48 & 2.42 & 3.80 & 6.00 & 8.93 & 10.95 & 9.56 \\
8 & 0.65 & 1.10 & 1.74 & 2.63 & 4.10 & 6.44 & 9.56 & 11.03 \\
\hline
\end{tabular}

sumed to be rawinsondes, with $u$ - and $v$-wind components and $\theta$ observed at each of the eight model levels. Observations and new analyses were generated every $12 \mathrm{~h}$, followed by a 12-h forecast with the QG model that generates the background at the next analysis time. Observations were generated by adding noise to truth run values of $u, v$, and $\theta$ at the observation locations. The noise was generated to be random yet consistent with observation-error covariance statistics, as in Houtekamer (1993). Actual observation-error covariances were adapted from the rawinsonde variances given in PD92 and used the vertical correlations given in Eq. (3.19) of Bergman (1979). Tables 1 and 2 provide observation-error covariances for potential temperature and winds, respectively. [Note that we have tested observation-error covariances with virtually no vertical correlations of errors, as in Hollingsworth and Lönnberg (1986) and Lönnberg and Hollingsworth (1986); though analysis errors decreased by effectively having more independent observations, most of the rest of the results we will describe were negligibly affected.]

The experiments were based on the three observational networks shown in Fig. 1: a low-density network (observations $\sim$ every $20^{2}$ grid points), a moderatedensity network ( every $10^{2}$ grid points), and a highdensity network ( every $5^{2}$ grid points). Observations locations were selected sequentially and randomly, using a one-dimensional Latin square algorithm (Press et al. 1992) that enforces a minimum distance between observations. The moderate-density network is a superset of the low-density network, and the high-density network a superset of the moderate. For simplicity, observations were located at the model grid points.

We shall focus primarily on a time series of 195 days of analyses and the 12-h background forecasts. This time period is substantially the same as the one used in the companion paper (SNY). So that samples from different analyses may be considered statistically independent, we will focus on a subset of 16 analyses sampled every 10 days after a 50-day spinup. For the low- and highdensity networks, $n_{e}=50$ independent cycles, or "members," were generated; for the moderate-density network, $n_{e}=200$ members were generated.

We will measure errors in three norms: the $L^{2}$ norm, the total-energy norm, and the pseudo-potential enstrophy norm. Given a geopotential perturbation from the 


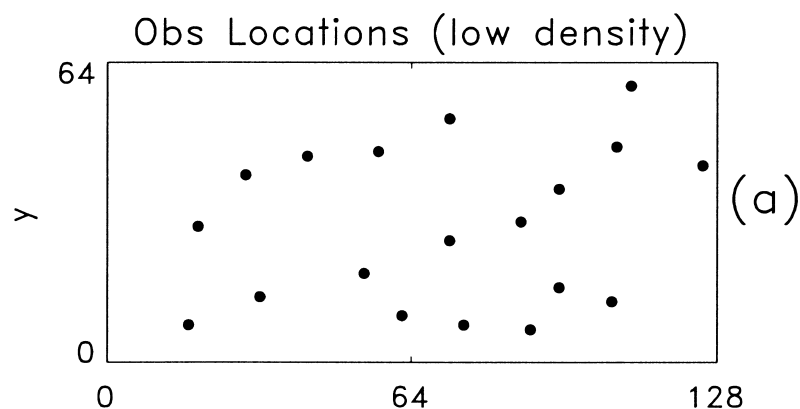

Obs Locations (moderate density)
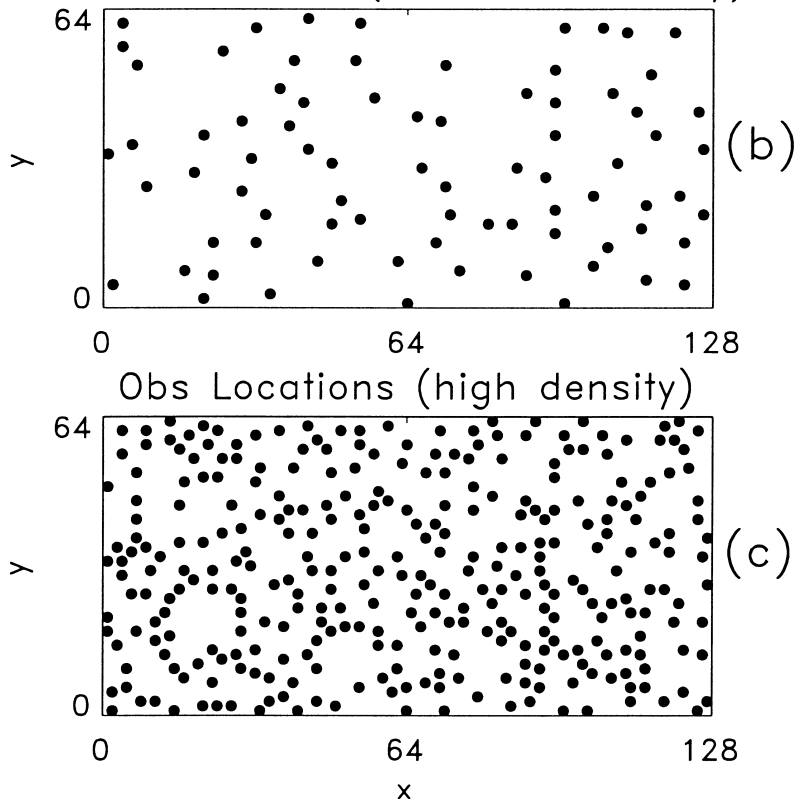

FIG. 1. Network of simulated rawinsondes used in this paper: (a) low-data-density network ( $\sim$ one obs every $20^{2}$ grid points), (b) moderate-data-density network ( $\sim$ one obs every $10^{2}$ grid points), and (c) high-data-density network ( $\sim$ one obs every $5^{2}$ grid points).

true state $\Phi^{\prime}$, a PV perturbation $q^{\prime}$, and $n$ grid points, the $L^{2}$ norm is defined as

$$
\|\cdot\|_{L^{2}}=g^{-1} n^{-1 / 2}\left[\sum_{j=1}^{n}\left(\Phi_{j}^{\prime}\right)^{2}\right]^{1 / 2} .
$$

Similarly, the total energy norm is defined as

$$
\begin{aligned}
\|\cdot\|_{\text {energy }}= & f^{-1} n^{-1 / 2} \\
& \times\left\{\sum_{j=1}^{n}\left[\left(\frac{\partial \Phi^{\prime}}{\partial x}\right)_{j}^{2}+\left(\frac{\partial \Phi^{\prime}}{\partial y}\right)_{j}^{2}+\left(\frac{f^{2}}{N^{2}} \frac{\partial \Phi^{\prime}}{\partial z}\right)_{j}^{2}\right]\right\}^{1 / 2},
\end{aligned}
$$

and the enstrophy norm is defined as

$$
\|\cdot\|_{\text {enstrophy }}=n^{-1 / 2}\left(\sum_{j=1}^{n} q_{j}^{\prime 2}\right)^{1 / 2} .
$$

Here, $N$ is the Brunt-Väisälä frequency, $g$ is the gravitational constant, and $f$ is the Coriolis parameter.
Hereafter, in our summaries of analysis-error statistics, the operator $\{\cdot\}$ denotes an average over all $n_{e}$ members; $\langle\cdot\rangle$ denotes an average over the subset $n_{d}=$ 16 cases, and - denotes a horizontal average over all grid points at a particular model level.

Our intent was to generate random samples of analysis errors given a knowledge of the true state of the atmosphere and a specific data assimilation system. To this end, independent, parallel data assimilation cycles were generated using classical Monte Carlo techniques (Press et al. 1992). Different initial conditions were used for each member of this ensemble, and a 12-h forecast was made for each member to the first analysis time. Thereafter, the following procedure was repeated for each member: 1) Generate a unique set of observations by adding noise to truth run fields, as previously described. 2) Update this member background forecast with the associated new set of observations using 3DVAR, described in the following section. 3) Make a forecast to the next analysis time, whence this forecast becomes the background for the next analysis cycle. This procedure is illustrated in Fig. 2. This produces an ensemble time series of analyses and short- range forecasts.

\section{Objective analysis}

A 3DVAR scheme following PD92 will be used here. This scheme as applied to the QG channel model is also described in more detail in Morss (1998, hereafter M98), Morss et al. (2001), and Hamill et al. (2000). Though more advanced and computationally expensive analysis schemes exist, such as four-dimensional variational data assimilation, or 4DVAR (e.g., Thépaut et al. 1993a,b), the 3DVAR algorithm is still used operationally at many centers worldwide and is appealing here because of its relative computational simplicity.

Let $m=$ number of observations and $n=$ number of forecast model variables, here 83850 . Hereafter, we generally follow the notational convention of Ide et al. (1997). Define H as the model operator, which transforms the forecast model variables to observation variables. In this problem, $\mathbf{H}$ is linear. Further, define $\mathbf{x}^{a}$ as the $n$-component analysis and $\mathbf{x}^{b}$ the model background state (also known as the prior, or "first guess"). Let $\mathbf{y}^{o}$ - Hx ${ }^{b}$ represent an $m$-component vector of observation increments (the difference between the observations and the transformed background variables at the observation locations). Let $\mathbf{B}$ be an estimate of the $n \times n$ forecasterror covariance matrix and $\mathbf{R}$ an $m \times m$ covariance matrix of the errors in the observations and representativeness, combined. Then, following Lorenc (1986) we can write a functional of the form

$$
\begin{aligned}
& J(\mathbf{x})=\frac{1}{2}\left[\left(\mathbf{x}-\mathbf{x}^{b}\right)^{\mathrm{T}} \mathbf{B}^{-1}\left(\mathbf{x}-\mathbf{x}^{b}\right)\right. \\
&\left.+\left(\mathbf{y}^{o}-\mathbf{H} \mathbf{x}\right)^{\mathrm{T}} \mathbf{R}^{-1}\left(\mathbf{y}^{o}-\mathbf{H} \mathbf{x}\right)\right] .
\end{aligned}
$$


Truth Run

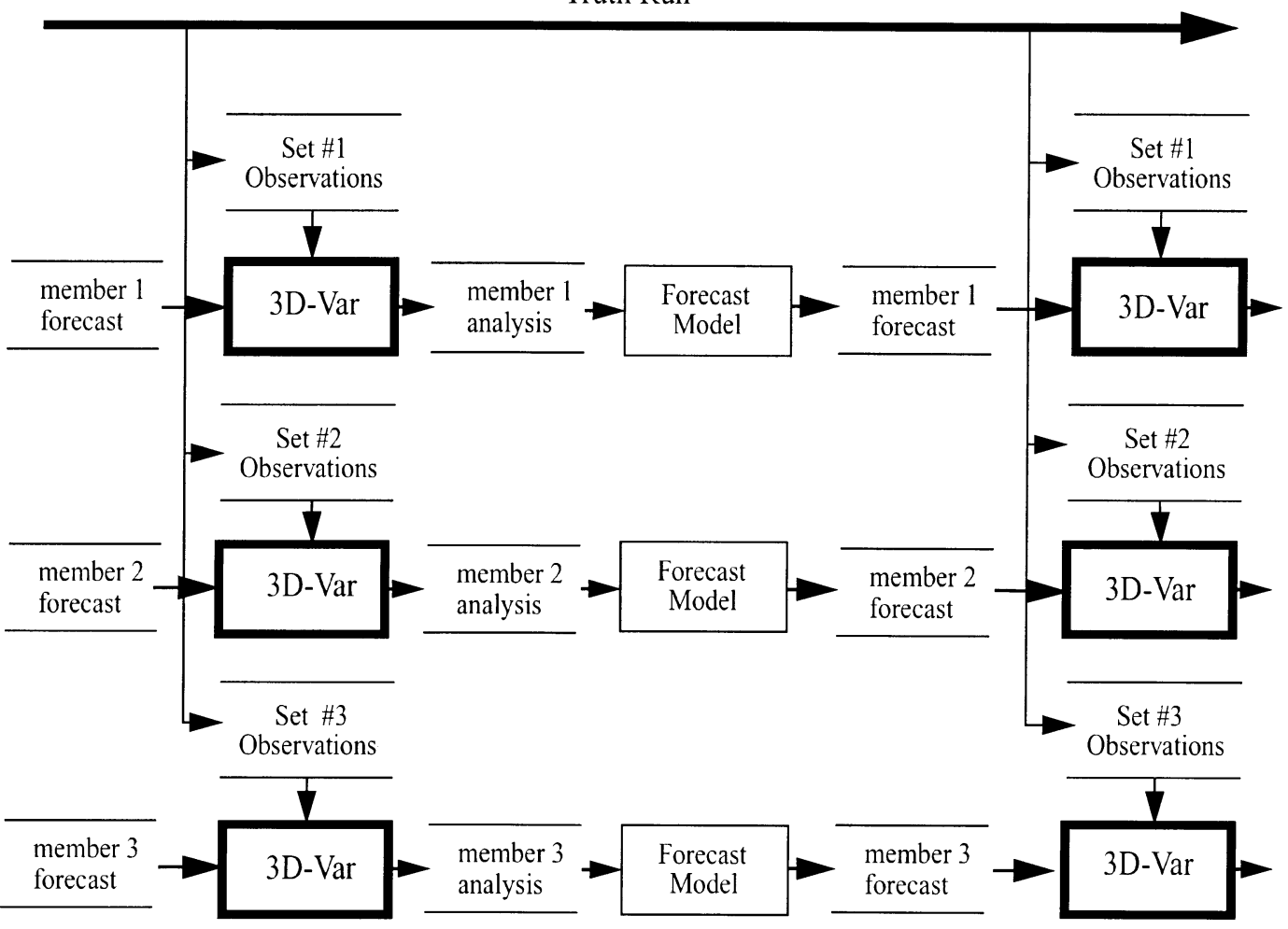

FIG. 2. Illustration of the method by which independent, parallel analyses are created. Different members are initialized with different background forecasts, and different sets of observations are assimilated into different members. Observations are created by adding random error to vertical profiles taken from the true model state; random errors are consistent with observation-error covariances. Member analyses are then used as initial conditions for a forecast to the next data assimilation time. The process is then repeated.

We seek the state $\mathbf{x}$ where the functional is a minimum; this is the optimal compromise state between the background and the new observations. By differentiating $J$ with respect to $\mathbf{x}$ and setting the result equal to zero, and then further multiplying through by $\mathbf{B}$ and rearranging terms we get

$$
\left(\mathbf{I}+\mathbf{B} \mathbf{H}^{\mathrm{T}} \mathbf{R}^{-1} \mathbf{H}\right)\left(\mathbf{x}^{a}-\mathbf{x}^{b}\right)=\mathbf{B} \mathbf{H}^{\mathrm{T}} \mathbf{R}^{-1}\left(\mathbf{y}^{o}-\mathbf{H} \mathbf{x}^{b}\right) .
$$

At each assimilation time, our implementation of 3DVAR solved (5) for the analysis increments $\mathbf{x}^{a}-\mathbf{x}^{b}$ using a conjugate residual descent algorithm (M98; Smolarkiewicz and Margolin 1994). The analysis increment was then added to the background to produce the analysis, which was used as the initial condition for the subsequent QG model forecast to the next analysis time.

Some assumptions were made to simplify the approximate background-error covariance matrix B. In 3DVAR, B was assumed (a) to be fixed in time, (b) to be diagonal in horizontal spectral coordinates, and (c) to have separable horizontal and vertical structures with simple vertical correlations. Assumptions a and b follow PD92; for assumption b, B was calculated as

$$
\mathrm{B}=\mathbf{S C S}^{\mathrm{T}} \text {, }
$$

where $\mathbf{S}$ is the transform from spectral space to grid points; $\mathbf{S}^{\mathrm{T}}$ is the adjoint operator of $\mathbf{S}$, which transforms from grid points to spectral coefficients; and $\mathbf{C}$ is the $n$ $\times n$ background-error covariance matrix in the Fourier basis. For computational efficiency, off-diagonal elements of $\mathbf{C}$ are set to 0 in the horizontal covariances. See M98 for more details on the parameterization of vertical correlations in this implementation of 3DVAR.

To optimize the data assimilation, horizontal forecast background-error variances in spectral space should be tuned to the (here, known) time-average backgrounderror statistics associated with each of the observational networks shown in Fig. 1. These background-error variances for each network density are plotted as a function of horizontal wavenumber (in units of horizontal waves per domain length) in Figs. 3a-c. Background-error covariances were derived through the iterative methodology described in M98.

\section{Results}

a. Spatial, temporal, and spectral characteristics of analysis errors

We first examine how analysis-error characteristics change with the observation density. Consider the time 

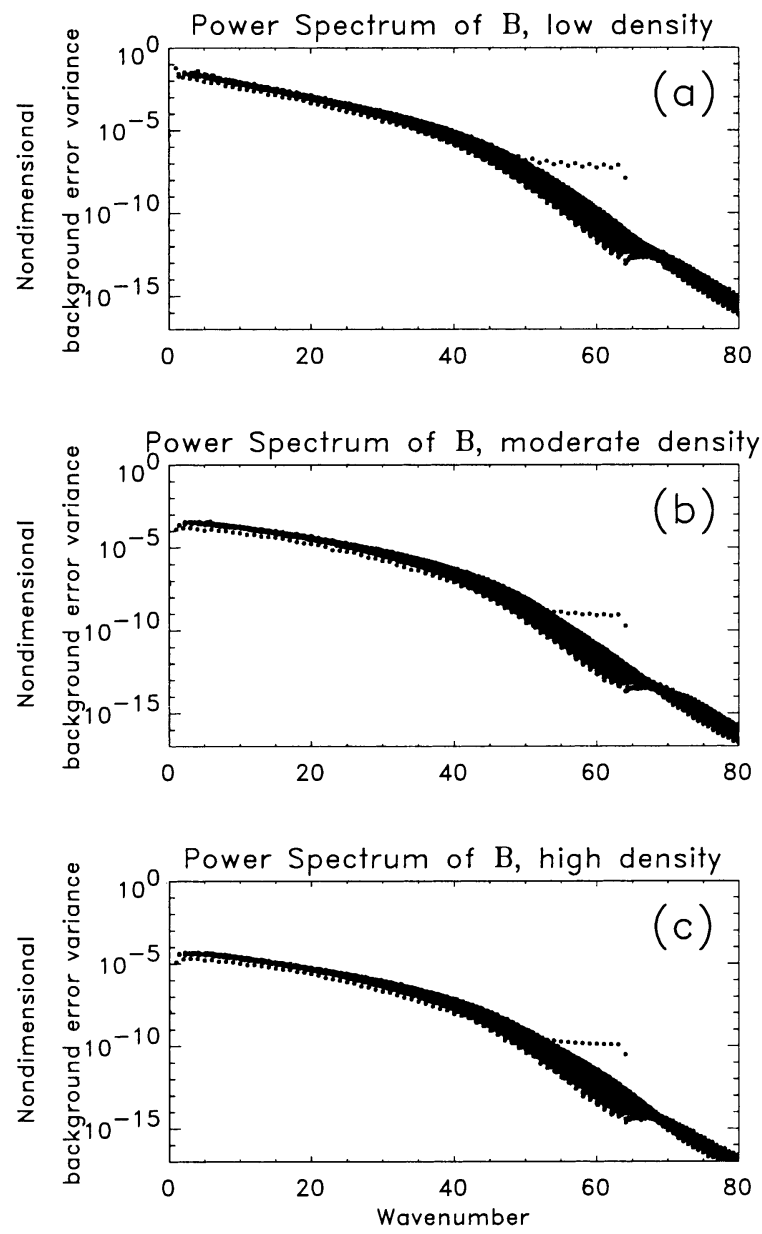

FIG. 3. Nondimensionalized background-error variances as a function of horizontal wavenumber for pseudo-potential vorticity at model level 4: (a) low-data-density network, (b) moderate-data-density network, and (c) high-data-density network.

series of analysis errors (Fig. 4). Analysis errors were computed with respect to the truth run solution and plotted for the $L^{2}$ norm, the total energy norm, and the pseudo-potential enstrophy norm as defined in Eqs. (1)(3). All norms exhibited similar qualitative behavior. In each norm, error generally decreased with increasing observational data density (see M98 for tests over a wider range of observation densities). However, note that the fourfold increase in observational data density between the low and moderate densities had a much greater effect than the increase from moderate to high data density. Qualitatively, this is consistent with the relationship between observational data density and error described in M98 and Morss et al. (2001). There, the authors demonstrated the existence of three regimes: 1) a regime where the observations were so sparse that new observations had little incremental benefit, 2) a regime where increasing the observational density provided a comparatively large benefit, and 3) a regime where observations were so dense that additional ob- servations were apparently providing redundant information and increasing the observation density was of little benefit. We appear to have spanned regime 2 between the low- and moderate-density observations but are in regime 3 between our tested moderate- and highdensity networks.

As in Morss et al. (2001), temporal variations in the analysis error in Figs. $4 \mathrm{a}-\mathrm{c}$ also tended to be larger when fewer observations were present; that is, the percent deviations of the error of any arbitrarily selected analysis from the time-averaged analysis error was typically larger, and periods of good or bad analyses lasted longer. The spatial variability of uncertainty across the domain at a given time was reduced as well when assimilating more observations. Let $\sigma_{i}^{\max }$ be the maximum standard deviation (spread) of streamfunction $\Phi$ over all grid points at model level 4 on the $i$ th case day of the 16 case days. Here spreads were calculated at each grid point with respect to the mean at that grid point of the 50 members. Similarly, let $\sigma_{i}^{\min }$ be the minimum spread across the domain, and $\bar{\sigma}_{i}$ be the horizontal average of spread over all grid points. Table 3 shows $\left\langle\left(\sigma_{i}^{\max }-\right.\right.$ $\left.\left.\sigma_{i}^{\min }\right) / \bar{\sigma}_{i}\right\rangle$, a nondimensional measure of the spatial variability of analysis uncertainty. The spatial variability of analysis uncertainty across the domain decreases as the number of observations increases.

We next examined vertical cross sections of error statistics. To do so, we selected on each case day a point at model level 4 where the spread among the background forecasts about their mean was large relative to nearby values. We then generated vertical cross sections aligned along the model level 4 streamline through the point. Using 50 members, we correlated the errors of all points along the cross section with the originally selected point at model level 4 , producing a vertical cross section of correlations $\mathbf{C}_{\mathbf{i}}$ for the $i$ th case day. In Figs. 5a,b, the $\left\langle\mathbf{C}_{\mathbf{i}}\right\rangle$ before and after the objective analysis is performed are shown for the moderate-density network. Notice the upshear tilt to the error structures in the background, suggesting these errors were projecting on baroclinically active structures. However, for the analysis in Fig. 5b, there was much less notable tilt in the correlation fields, showing how the analysis modifies prior dynamical structure. Cross sections were quite similar for the lowand high-density networks (not shown).

Let $\mathbf{E}_{T}, \mathbf{E}_{K}$, and $\mathbf{E}_{P}$ represent a state vector of total, kinetic, and potential energy errors. Here, $\mathbf{E}_{T}=\mathbf{E}_{K}+$ $\mathbf{E}_{P}$, and kinetic and potential energies at the $j$ th grid point are defined by

$$
\begin{aligned}
& \mathbf{E}_{K_{j}}=f^{-1} \times\left\{\left(\frac{\partial \Phi^{\prime}}{\partial x}\right)_{j}^{2}+\left(\frac{\partial \Phi^{\prime}}{\partial y}\right)_{j}^{2}\right\}, \text { and } \\
& \mathbf{E}_{P_{j}}=\frac{f}{N^{2}}\left(\frac{\partial \Phi^{\prime}}{\partial z}\right)_{j}^{2} .
\end{aligned}
$$

Figures 6a,b show $\left\{\left\langle\overline{\mathbf{E}}_{T}\right\rangle\right\},\left\{\left\langle\overline{\mathbf{E}}_{K}\right\rangle\right\}$, and $\left\{\left\langle\overline{\mathbf{E}}_{P}\right\rangle\right\}$ as a function of vertical level in the model. Errors in energy 

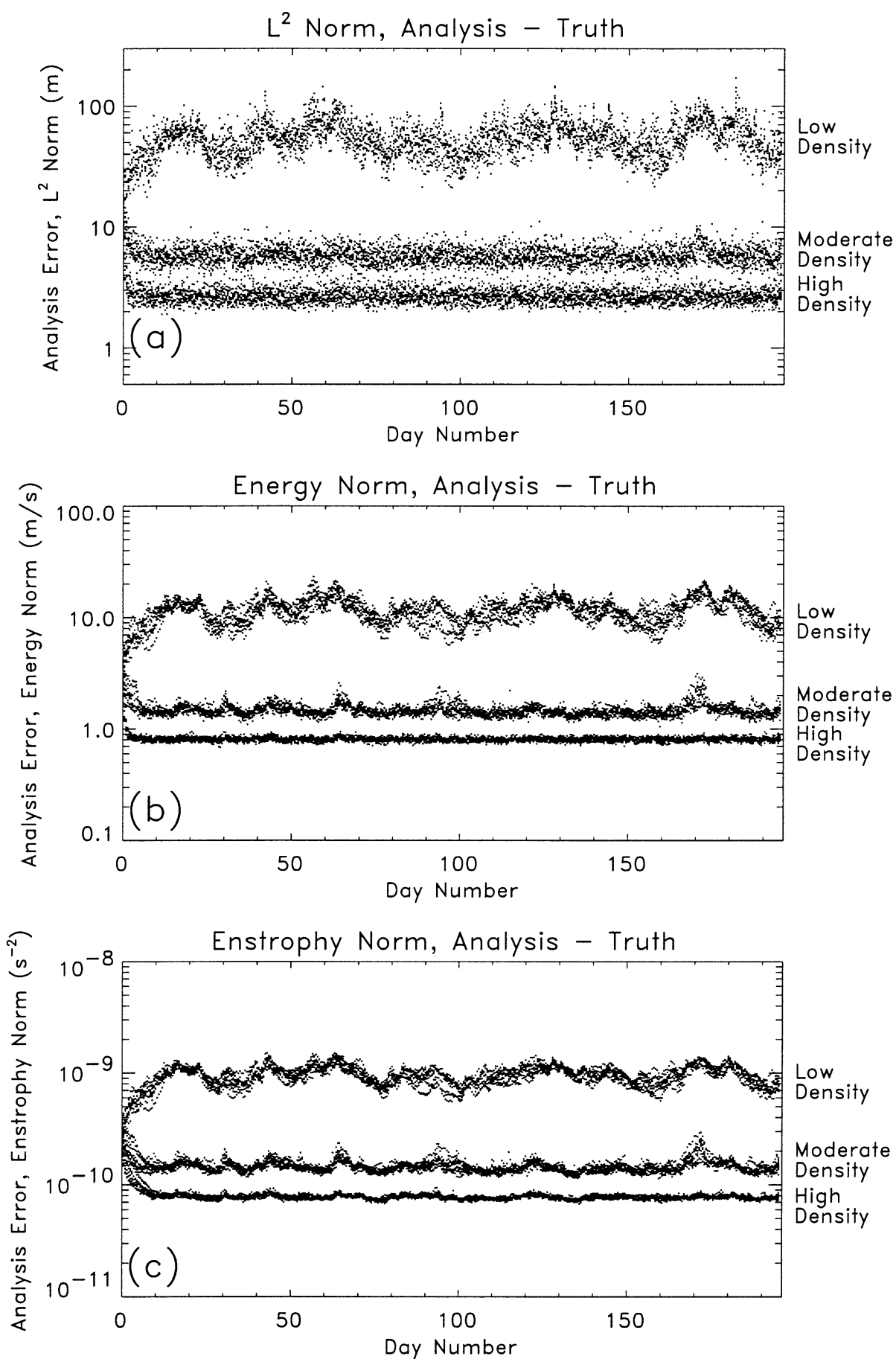

FIG. 4. Time series of analysis errors. Each dot represents the error of 1 member of a 50-member ensemble of analyses, valid at the specified time. (a) Error in the $L^{2}$ norm; (b) error in the total-energy norm, and (c) error in the pseudo-potential enstrophy norm.

were nearly $10 \times$ higher at the top model level $(\sim 270$ $\mathrm{hPa}$ ) than they were at the third model level $(\sim 650 \mathrm{hPa})$. The energy profiles were not much different in character between the background and analysis. This result is consistent with previous error studies (e.g., Hollingsworth and Lönnberg 1986; Rabier et al. 1998; Barkmeijer et al. 1998, 1999), which document that background and analysis wind errors at $300 \mathrm{hPa}$ are typically much larger near the tropopause than in the low to middle troposphere. This suggests that the singular-vector perturbations for ensemble forecasts measured in the totalenergy initial norm, perturbations that are typically larg- 
Moderate density, avg. background $X$-section of correlation

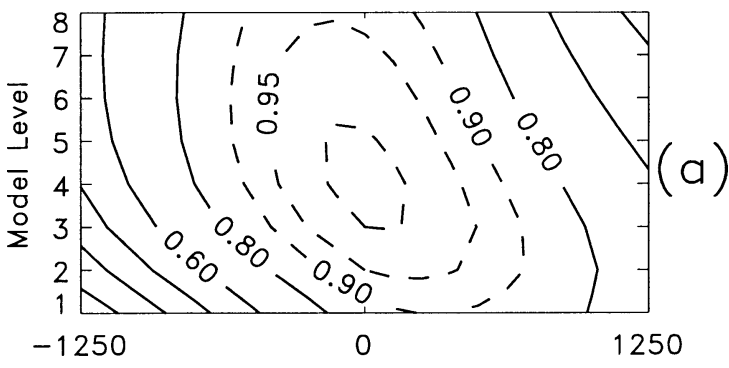

Moderate density, avg. analysis $X$-section of correlation

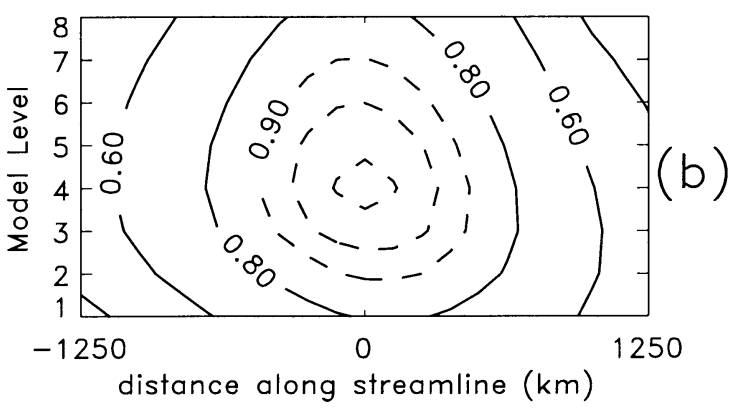

FIG. 5. Composite cross section of correlations of member deviations from the ensemble mean, with the cross section taken along a streamline through a local maxima of $\sigma(\phi)$ at model level 4. Dashed contours at 0.90, 0.95, and 0.99 correlation. (a) Background, moderate-density network; (b) analysis.

est in the low to midtroposphere, are generally not consistent with the analysis-error covariances. This is perhaps better illustrated in Fig. 7, a vertical profile of the average background and analysis enstrophy errors $\left\{\left\langle\overline{q^{\prime 2}}\right\rangle\right\}$, for the moderate-density network, here non-
TABLE 3. Values for $\left\langle\left(\alpha_{i}^{\max }-\alpha_{i}^{\min }\right) / \bar{\alpha}_{i}\right\rangle$ as a function of data density and analysis or background.

\begin{tabular}{lcc}
\hline \hline & Background & Analysis \\
\hline Low density & 1.84 & 1.59 \\
Moderate density & 1.29 & 1.13 \\
High density & 1.02 & 0.67 \\
\hline
\end{tabular}

dimensionalized as in SNY. Perturbations that are initially white in energy will also have a uniform vertical profile of enstrophy (SNY); as shown here, the enstrophy errors are much larger at the top and bottom lids than in the domain interior.

The horizontal spectrum of errors is also of interest. Let $F\left(\mathbf{E}_{T}\right)$ represent an average Fourier transform of the total energy at model level 4 in the zonal direction for grid rows in the center third of the channel. Figure 8 plots $\left\{\left\langle F\left(\mathbf{E}_{T}\right)\right\rangle\right\}$. For the low-density network, errors are saturated beyond wavenumber 20. Coincidentally, Daley and Mayer (1986) found a similar result with circa 1979 global analyses where no useful information was provided beyond wavenumber 20 . Note that in our experiment, there does appear to be useful information out to wavenumber 64 for the moderate- and high-density networks. Even though the observation density is not sufficient to resolve these scales, the information supplied by the background, including small-scale features organized by the large-scale dynamics, apparently survive the analysis process, a sign that dynamical structure that is organized during the forecast tends to be preserved in the analysis.

\section{b. Organization of errors by forecast dynamics}

A well-known property of chaotic dynamical systems is that the volume element defined by almost any set of
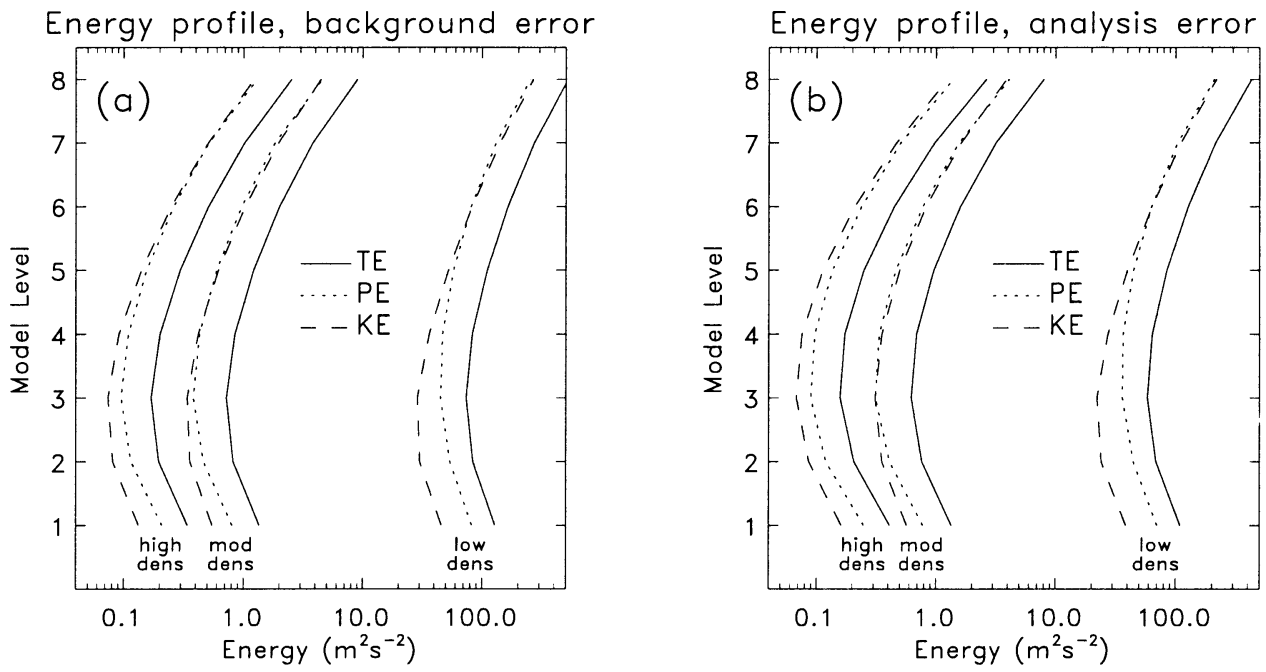

FIG. 6. Vertical profiles of total, kinetic, and potential energies of analysis and background errors, derived from an average over all case days and ensemble members. (a) Error in the background; (b) error in the analysis. 


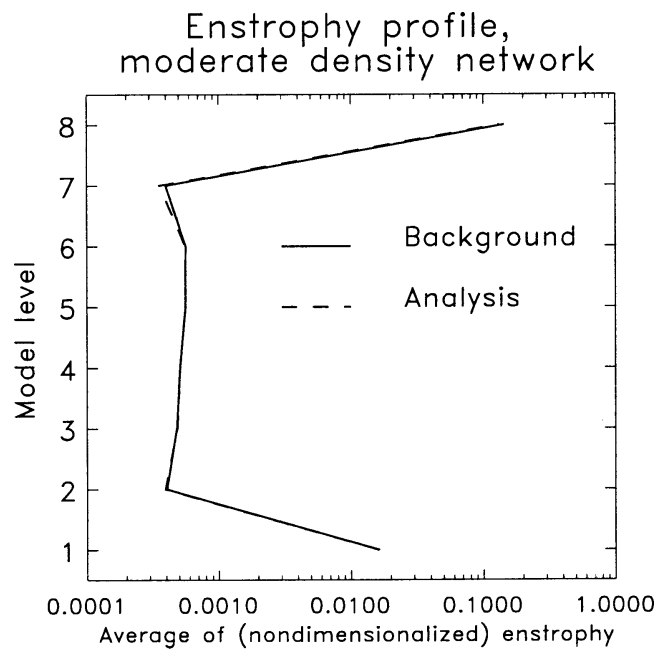

FIG. 7. Vertical profiles of enstrophy error for a moderate-density network, averaged over the domain and over all case days, for the analysis and the background.

$n_{l}$ independent, infinitesimal perturbations converges over time to the subspace defined by first $n_{l}$ (backward) Lyapunov vectors or LVs (Legras and Vautard 1996). We have calculated the leading $20 \mathrm{LVs}$ for this model, normalizing the LVs to be orthogonal in a total-energy sense. SNHA discuss the methodology for calculation of the Lyapunov vectors, and SNY show that for this model, an ensemble of perturbations constructed to be initially white in energy projects very significantly $(\sim 0.7)$ on the subspace of the leading 20 Lyapunov vectors after 2 days, indicating the quick manner in which dynamics organizes the errors. Here we seek to determine the extent to which background and analysis errors project on the Lyapunov vectors. This will provide some indication of how much dynamical structure is in the background and how much this information is modified in the analysis process.

Let $\mathbf{p}_{b}$ and $\mathbf{p}_{a}$ represent, respectively, a 20-dimensional vector of projections of background and analysis errors onto the leading $20 \mathrm{LVs}$, for a given time and ensemble member. Figure 9 shows $\left\{\left\langle\mathbf{p}_{b}\right\rangle\right\}$ and $\left\{\left\langle\mathbf{p}_{a}\right\rangle\right\}$ for each of the three networks. Notice that the analysis process reduces the average projection, yet a substantial projection remains after the analysis. Comparing the projections for the different networks, the projections of background error were rather similar for the low- and moderate-density network, but are smaller for the highdensity network. Normally, one might expect that the fewer the observations that were assimilated, the less randomization of the dynamical structure and, hence, the larger the projection onto the leading LVs. We hypothesize that this effect is being counterbalanced by the stronger nonlinearities due to the higher errors associated with the low-density network. However, for the subsequent analysis, there was less and less projection as observation density increased. Note also that there was some positive correlation between the amount of

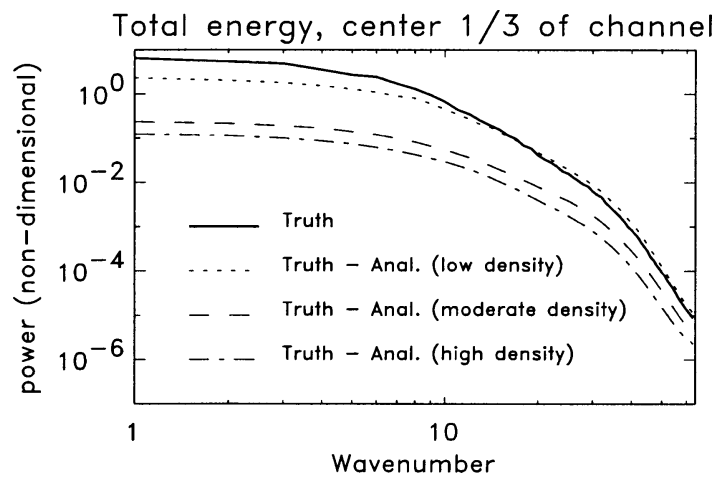

FIG. 8. Total-energy power spectra for the truth run and for analysis errors, averaged over east-west cross sections in the center $1 / 3$ of the channel, over case days, and over ensemble members.

projection and the LV number, with more projection onto the rapidly growing directions (associated with the lower Lyapunov vector numbers).

The dynamical structure in the background-forecast errors tended to be destroyed in the assimilation process, yet those errors are on average drawn back by the forecast dynamics toward the subspace spanned by the leading LVs. What are the characteristics of errors in this subspace? SNHA show that the potential vorticity of the leading LVs is strongly related to $\|\nabla \bar{q}\|$, the magnitude of the gradient of the mean potential vorticity. Given an ensemble of initial errors, this characteristic of the LVs manifests as a tendency for the variance of the potential vorticity errors to correlate spatially with $\|\nabla \bar{q}\|$ as the ensemble evolves (SNY). This property emerges quickly regardless of the initial error structure. Does this property survive in a cycled analysis system? Here, for each network, we have determined $\langle\operatorname{Corr}[\sigma(q)$, $\|\nabla \bar{q}\|]\rangle$, an average correlation of the spread of PV with its gradient at the top and bottom lids. The correlation is the average over the 16 case days and is computed using the model grid points in the center two-thirds of the channel. The correlations are $0.56,0.61$, and 0.58 for background forecasts for the low-, moderate-, and high-density networks. The corresponding correlations for the analysis are $0.49,0.47$, and 0.29 . Again, more dynamical structure is lost during the analysis process when the observation density is higher, but even at the highest density, not all structure is lost. Further, one could think about the process in reverse; the analysis serves as the initial condition for the next background forecast, so whatever dynamical structure is lost during the analysis is quickly reestablished.

\section{c. Information content and relation to ensemble size}

We next seek to understand how the variance characteristics of an ensemble will change with the size of that ensemble. This has implications for ensemble-based data assimilation approaches. If adding more ensemble members provides only marginally more detail on the 


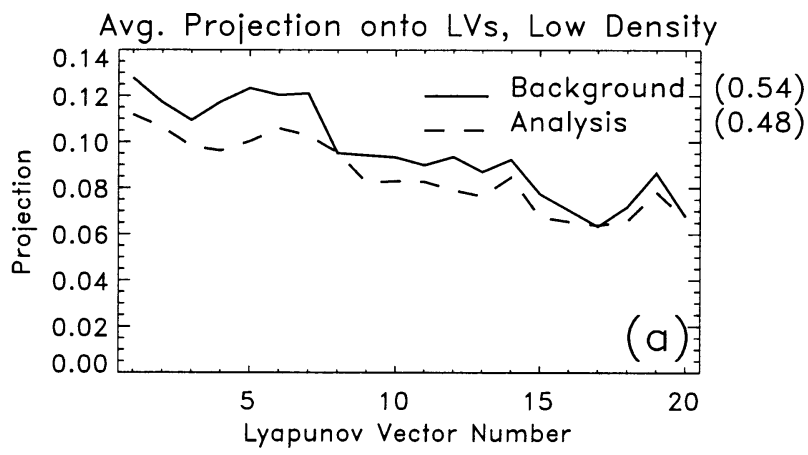

Avg. Projection onto LVs, Moderate Density
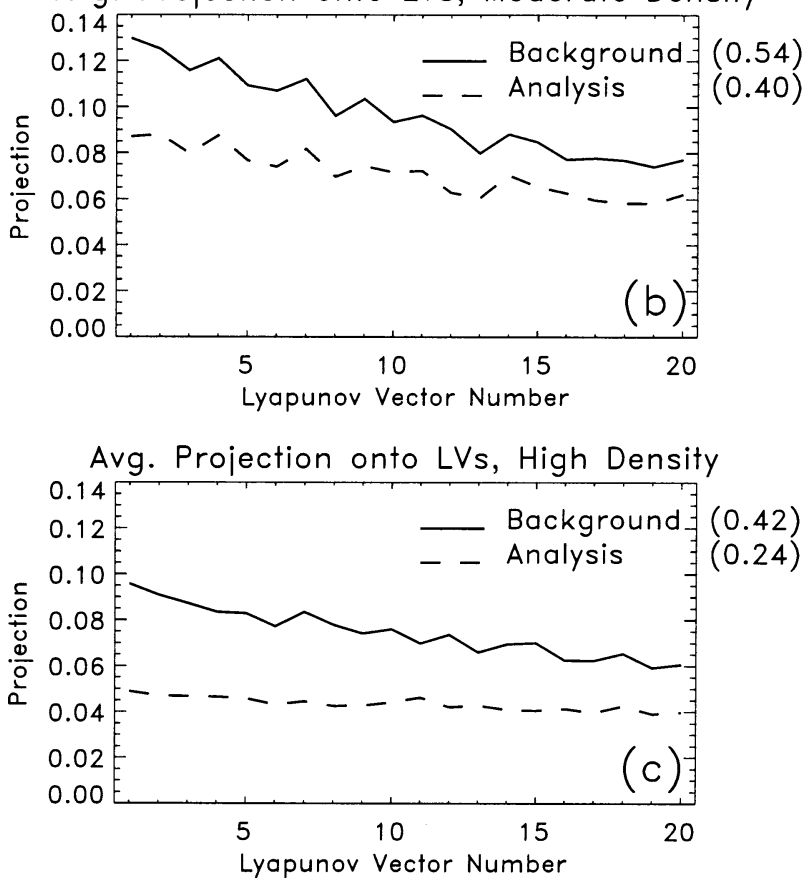

FIG. 9. Time- and ensemble-average projection of errors onto the leading 20 Lyapunov vectors: (a) low-density network, (b) moderatedensity network, and (c) high-density network.

structure of errors, then these added members may not be worth the extra computation.

To begin to understand the variance characteristics, let us transform a state vector $\mathbf{x}$ with an operator $\mathbf{T}$ that converts it into a vector $\mathbf{e}$ of nondimensional winds and temperatures, $\mathbf{e}=\mathbf{T} \mathbf{x}$, such that the standard dot product is an energy norm. We wish to determine the eigenvalues and empirical orthogonal functions (EOFs) of the covariance matrix estimated from the ensemble in a total energy norm: $\hat{\mathbf{P}}_{e}=1 /\left(n_{e}-1\right) \mathbf{E} \mathbf{E}^{\mathbf{T}}$, where $\mathbf{E}=\left(\mathbf{e}_{1}^{\prime}, \ldots, \mathbf{e}_{n_{e}}^{\prime}\right)$ and $\mathbf{e}_{i}^{\prime}=\mathbf{e}_{i}-\{\mathbf{e}\}$, that is, the $i$ th member's deviation from the ensemble mean. These calculations were accomplished by performing a singular value decomposition of $\mathbf{E}$. The resulting singular values and vectors can readily be shown to correspond to the square root of the eigenvalues and to the EOFs of $\hat{\mathbf{P}}_{e}$, respectively. With the vector of sorted eigenvalues $\lambda$ of $\hat{\mathbf{P}}_{e}^{f}$, we determined the expected percent
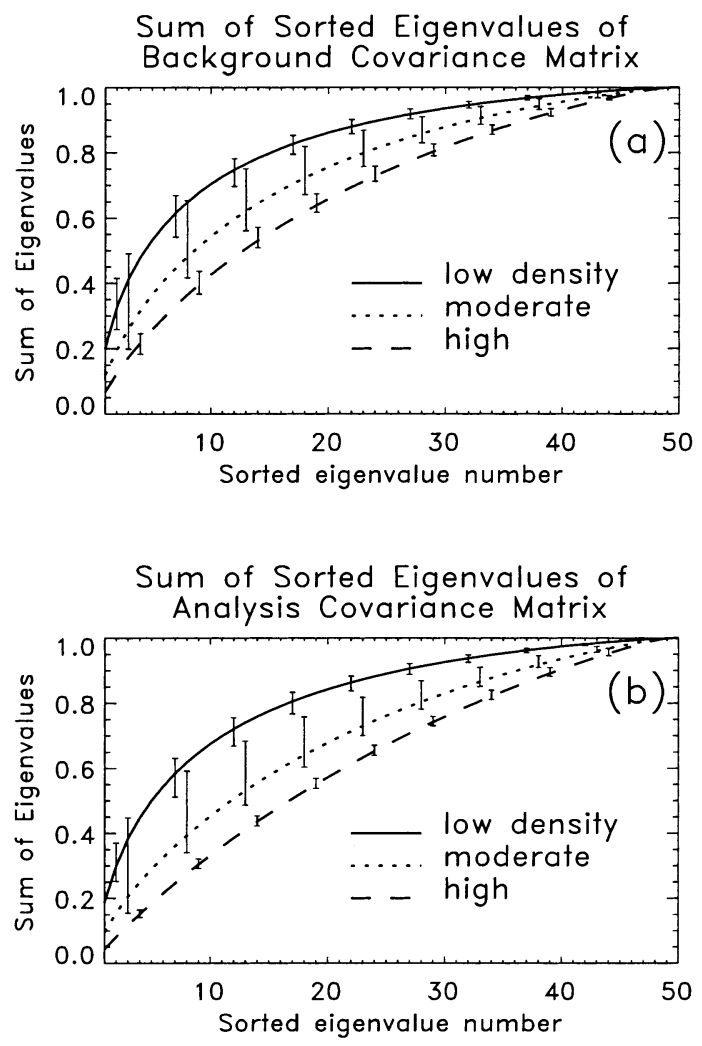

FIG. 10. Sum of leading eigenvalues of forecast/analysis covariance matrix divided by the trace of the matrix (=total sum of all eigenvalues). Lines represent average over all case days, and error bars indicate the smallest and largest from the 16-case-day sample. (a) Background; (b) analysis.

of the total variance in a $n_{e}$-member ensemble accounted for by the first $r$ EOFs of $\hat{\mathbf{P}}_{e}$; that is, $\Lambda_{r}=\sum_{i=1}^{r} \lambda_{i} / \sum_{i=1}^{n_{e}} \lambda_{i}$. Figures 10a,b show $\left\langle\Lambda_{r}\right\rangle$ as a function of $r$ for the three observational densities and for both the analysis and background. Error bars represent the minimum and maximum from the 16-day subsample. Note that the initial steepness of the eigenvalue spectrum decreased with increasing observational data density. Also note that the analysis curves in Fig. 10b were slightly flatter than the background curves in Fig. 10a.

We expect that the spectrum flattening can be explained by two effects. First, observations have random errors. To the extent that the structure of backgrounderror covariances permit analysis increments to be introduced in directions off the attractor, the noise of the observations can introduce perturbations in new directions orthogonal to that organized by the forecast dynamics. The more observations, the greater this effect. However, even if the background-error covariances are correctly modeled so they are much larger in magnitude in the directions of the leading Lyapunov vectors, the analysis process can flatten the spectrum. In this case, the analysis corrections will be making larger corrections in these growing directions, and the error variance will be concomitantly reduced, flattening the spectrum. 
The prior effect is undesirable, increasing analysis errors, while the latter effect is desirable, decreasing the error. It is difficult to determine how much of the spectrum flattening is caused by each effect.

The curves in Fig. 10 provide some indication of the relative value of additional ensemble members in specifying the variance but do not provide quantitative information on the size of the ensemble needed to account for a specified fraction of the variance. Here, we examine the steepness of the covariance spectrum in a slightly different manner. We first constructed covariance matrices for ensembles of various sizes from 5 to 200 members. Figure 11a shows time-averaged eigenvalue spectra of $\langle\lambda\rangle$ of $\hat{\mathbf{P}}_{e}^{f}$ for a 200 -member ensemble. The curves were produced for the moderate density network in Fig. 1b. The eigenvalue spectra are initially quite steep. Now, given an $n_{e}$-member ensemble, how many of the leading EOFs are necessary to account for an expected percentage of the variance, and how does this number of EOFs change as $n_{e}$ changes? Figures $11 \mathrm{~b}, \mathrm{c}$ show the average number of EOFs it takes to account for $25 \%, 50 \%, 75 \%$, and $90 \%$ of the total variance for various sized ensembles. For example, Fig. 11 b suggests that for a 100-member ensemble, the leading 50 EOFs will account for $90 \%$ of the background variance, but approximately 81 EOFs are needed to account for the same percentage in the 200-member ensemble. Figure 11c shows that the number of members needed is greater for the analysis compared to the background, again suggesting the objective analysis tends to randomize the prior, low-dimensional structure of background errors. The curve appears almost flat at 25 percent variance by 200 members, suggesting that an ensemble more quickly determines accurate leading EOFs than smaller EOFs, a convergence property typical in EOF analysis (e.g., Preisendorfer 1988; Wilks 1995).

Along similar lines, another interesting question is whether two ensembles of background forecasts of the same size will span the same dynamically relevant subspace. If errors in the forecasts project primarily in a low-dimensional subspace, then two random ensembles ought to span similar subspaces. To determine the extent of this similarity, the 200-member ensemble generated for the medium-density network was split into two groups of 100 members. For each of the 16 case days, the leading eigenvalues and EOFs of the covariance matrix for each group were determined. Figure 12 shows $\left\langle\mathbf{p}_{b}\right\rangle$, the 16-case-day average projection of sorted eigenvectors from ensemble members 1-100 of a 200member ensemble onto the subspace spanned by members 101-200. This is very similar to the subspace similarity index proposed by Buizza (1994). The leading EOF from one 100-member group projects about $95 \%$ onto the subspace of the other 100-member group, with lesser projections for subsequent eigenvectors. Note that even the trailing EOFs still have significant projection into a subspace spanned by an independent sample.
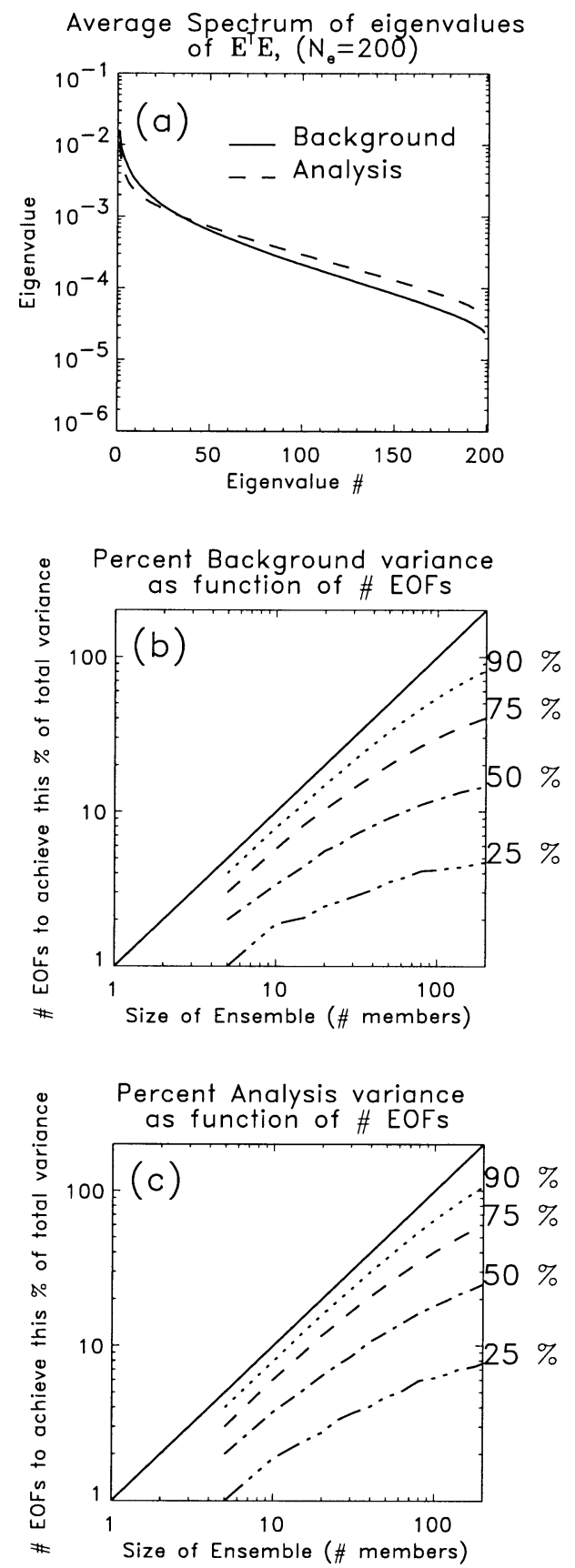

FIG. 11. (a) Time-average eigenvalue spectra of covariance matrix developed from a 200-member ensemble in an energy norm using the moderate-density network. (b) Number of EOFs it takes to account for prespecified percentage of background variance as a function of ensemble size. (c) As in (b) but for analysis variance.

\section{Conclusions}

We have conducted a set of experiments with a quasigeostrophic channel model and a 3DVAR assimilation system to examine some statistical and dynamical characteristics of analysis errors. The simulations were carried out by conducting a long time series of parallel data 


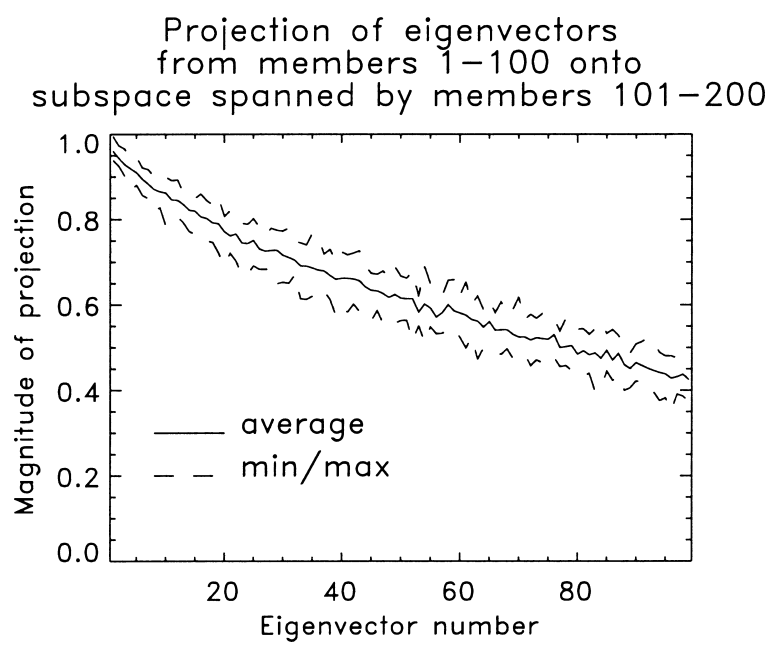

FIG. 12. Time-averaged projection of sorted eigenvectors from ensemble members 1-100 background forecasts of a 200 -member ensemble onto the subspace spanned by member background forecasts 101-200. Solid line indicates average over 16 case days; dashed lines indicate minimum and maximum over 16 case days.

assimilations, where different, equally likely observations were assimilated by different members. Three networks of observations were tested: low-, moderate-, and high-density networks.

Results reinforce previous findings of a nonlinear relationship between observational data density and analysis error; there was much more error reduction from low density to moderate than from moderate to high. Generally, the more observations, the more homogeneous the errors were in time and space. From low to moderate, the primary energy-containing larger scales of motion changed from being poorly analyzed to being much better analyzed. From moderate to high, the improvement was roughly similar across all scales of motion. Similarly, the wavenumber at which the noise (the power of the errors) exceeded the signal (the power in the true state) increased with increasing data density; higher wavenumbers were more effectively analyzed with increasing data density. As noted in other studies, errors were also much larger in the upper troposphere than in the middle troposphere. Vertical profiles of the enstrophy error showed that the errors were primarily at the model top and bottom lids, with little potential vorticity error on the interior. Taken together, these results suggest that unless observation density is uniformly high, background-error statistics should not be expected to be either stationary or isotropic. These results also suggest that background-error covariances differ from those that would be implied by a total-energy norm, and that total-energy singular vector perturbations for ensemble forecasts would be substantially different in structure from analysis-error covariance singular vectors.

Errors in the analysis showed characteristics of both the structured errors created by the chaotic dynamics as well as random observational errors. The correction of the background toward the observations reduced the error but also randomized the prior dynamical structure of the errors. We have shown here that the variance of the potential vorticity analysis at a grid point was strongly related to the magnitude of the potential vorticity gradient at that location. This characteristic was inherited from the forecast dynamics and persists in the analysis despite the effects of randomization.

The dynamical structure of errors depended on the observational data density: the higher the density of observations, the less the projection onto the leading backward Lyapunov vectors, and the more ensemble members were needed to capture a given percentage of the total analysis variance. However, even at the highest observational density, the analysis errors retained considerable dynamical structure.

One perhaps surprising result was how few ensemble members were needed to capture a large percentage of the overall analysis-error covariance structure. Many authors have previously suggested that Monte Carlo methodologies such as the one employed here are not particularly useful because an extraordinarily large number of members must be employed to span the interesting dynamics. Though a precise count of ensemble members would certainly be model and data assimilation specific, these results suggest that Monte Carlo methodologies are viable; here, a 100-member ensemble was able to span most of the important dynamics in a model with $O\left(10^{5}\right)$ degrees of freedom. This conclusion is of course supported by the growing body of evidence about the efficacy of Monte Carlo methodologies for ensemblebased data assimilation, as discussed in the introduction.

How much can these results be generalized across different models, different analysis systems, and under different assumptions (e.g., with model errors)? Though model errors may contribute to less dynamically structured error, we believe most of the conclusions are robust. Conclusions about the reduced dimensionality of analysis error may differ significantly with the model and analysis scheme. The larger the model state dimension and the more scales of motion resolved, the higher the expected dimensionality of both forecast and analysis error. On the other hand, with a better data assimilation system, such as one using flow-dependent background-error covariances, the increased use of dynamically structured error covariances should reduce the amount of randomization in the analysis by lessening off-attractor analysis increments. This may reduce the effective dimensionality of analysis errors.

To date, only the work of Barkmeijer et al. (1998, 1999) has explored how to generate an ensemble of initial conditions that grow rapidly yet are consistent with (time averaged) analysis-error statistics. These "Hessian" singular vectors were shown to be somewhat less useful for making probabilistic forecasts than their operational "total energy" singular vectors, perhaps because the analysis-error statistics were not flow depen- 
dent. The results presented here and in the companion papers (SNY, SNHA), as well as many results from ensemble data assimilation studies, strongly suggest that analysis-error statistics are flow dependent. Though theory indicates that ensemble initial conditions should be consistent with the analysis-error statistics, perhaps the generation of perturbations that are consistent with these time-averaged statistics can be quite inconsistent with the instantaneous analysis-error statistics. We hope to explore this in our future research.

Acknowledgments. This research was supported by the NCAR USWRP Science Program. The bulk of the research was carried out while the lead author was in NCAR's Advanced Studies Program. The lead author appreciates the support of the NOAA-CIRES Climate Diagnostics Center in finishing this work.

\section{REFERENCES}

Anderson, J. L., 1996: Selection of initial conditions for ensemble forecasts in a simple perfect-model framework. J. Atmos. Sci., 53, 22-36.

_ 2001: An ensemble adjustment Kalman filter for data assimilation. Mon. Wea. Rev., 129, 2884-2903.

— the nonlinear filtering problem to produce ensemble assimilations and forecasts. Mon. Wea. Rev., 127, 2741-2758.

Barkmeijer, J., M. van Gijzen, and F. Bouttier, 1998: Singular vectors and estimates of the analysis error covariance metric. Quart. J. Roy. Meteor. Soc., 124, 1695-1713.

_ R. Buizza, and T. N. Palmer, 1999: 3DVar Hessian singular vectors and their potential use in the ECMWF ensemble prediction system. Quart. J. Roy. Meteor. Soc., 125, 2333-2351.

Bergman, K. H., 1979: Multivariate analysis of temperatures and winds using optimum interpolation. Mon. Wea. Rev., 107, 14231444.

Bouttier, F., 1994: A dynamical estimation of forecast error covariances in an assimilation system. Mon. Wea. Rev., 122, 23762390.

Buizza, R., 1994: Sensitivity of optimal unstable structures. Quart. J. Roy. Meteor. Soc., 120, 429-451.

Burgers, G., P. J. van Leeuwen, and G. Evensen, 1998: Analysis scheme in the ensemble Kalman filter. Mon. Wea. Rev., 126, 1719-1724.

Daley, R., and T. Mayer, 1986: Estimates of global analysis error from the global weather experiment observational network. Mon. Wea. Rev., 114, 1642-1653.

Ehrendorfer, M., and J. J. Tribbia, 1997: Optimal prediction of forecast error covariances through singular vectors. J. Atmos. Sci., 54, 286-313.

Evensen, G., 1994: Sequential data assimilation with a nonlinear quasigeostrophic model using Monte Carlo methods to forecast error statistics. J. Geophys. Res., 99 (C5), 10 143-10 162.

_ , and P. J. van Leeuwen, 1996: Assimilation of Geosat altimeter data for the Agulhas current using the ensemble Kalman filter with a quasigeostrophic model. Mon. Wea. Rev., 124, 85-96.

Hamill, T. M., and C. Snyder, 2000: A hybrid ensemble Kalman filter3D variational analysis scheme. Mon. Wea. Rev., 128, 29052919.

— , and — 2002: Using improved background-error covariances from an ensemble Kalman filter for adaptive observations. Mon Wea. Rev., 130, 1552-1572.

- — , and R. E. Morss, 2000: A comparison of probabilistic forecasts from bred, singular vector, and perturbed observation ensemble forecasts. Mon. Wea. Rev., 128, 1835-1851.
- J. S. Whitaker, and C. Snyder, 2001: Distance-dependent filtering of background error covariance estimates in an ensemble Kalman filter. Mon. Wea. Rev., 129, 2776-2790.

Hansen, J. A., and L. A. Smith, 2001: Probabilistic noise reduction. Tellus, 53A, 585-598.

Heemink, A. W., M. Verlaan, and A. J. Segers, 2001: Variance-reduced ensemble Kalman filtering. Mon. Wea. Rev., 129, 17181728.

Hollingsworth, A., and P. Lönnberg, 1986: The statistical structure of short-range forecast errors as determined from radiosonde data. Part I: The wind field. Tellus, 38A, 111-136.

Houtekamer, P. L., 1993: Global and local skill forecasts. Mon. Wea. Rev., 121, 1834-1846.

_- and J. Derome, 1995: Methods for ensemble prediction. Mon. Wea. Rev., 123, 2181-2196.

- and H. L. Mitchell, 1998: Data assimilation using an ensemble Kalman filter technique. Mon. Wea. Rev., 126, 796-811.

_ , and _ 2001: A sequential ensemble Kalman filter for atmospheric data assimilation. Mon. Wea. Rev., 129, 123-137.

-, L. Lefaivre, and J. Derome, 1996: The RPN ensemble prediction system. Proc. ECMWF Seminar on Predictability, Vol II, Reading, United Kingdom, ECMWF, 121-146. [Available from ECMWF, Shinfield Park, Reading, Berkshire RG2 9AX, United Kingdom.]

Ide, K., P. Courtier, M. Ghil, and A. C. Lorenc, 1997: Unified notation for data assimilation: Operational, sequential, and variational. $J$. Meteor. Soc. Japan, 75, 181-189.

Ingleby, N. B., 2001: The statistical structure of forecast errors and its representation in the Met. Office global 3-D variational data assimilation scheme. Quart. J. Roy. Meteor. Soc., 127, 209-231.

Keppenne, C. L., 2000: Data assimilation into a primitive equation model with a parallel ensemble Kalman filter. Mon. Wea. Rev., 128, 1971-1981.

Legras, B., and R. Vautard, 1996: A guide to Lyapunov vectors. Proc. ECMWF Seminar on Predictability, Vol. I, Reading, United Kingdom, ECMWF, 1, 143-156. [Available from ECMWF Library, Shinfield Park, Reading, Berkshire RG2 9AX, United Kingdom.]

Leith, C. E., 1974: Theoretical skill of Monte Carlo forecasts. Mon. Wea. Rev., 102, 409-418.

Lermusiaux, P. F. J., and A. R. Robinson, 1999: Data assimilation via error subspace statistical estimation. Part I: Theory and schemes. Mon. Wea. Rev., 127, 1385-1407.

Lönnberg, P., and A. Hollingsworth, 1986: The statistical structure of short-range forecast errors as determined from radiosonde data. Part II: The covariance of height and wind errors. Tellus, 38A, 137-161.

Lorenc, A. C., 1986: Analysis methods for numerical weather prediction. Quart. J. Roy. Meteor. Soc., 112, 1177-1194.

_- , and Coauthors, 2000: The Met. Office global three-dimensional variational data assimilation scheme. Quart. J. Roy. Meteor. Soc., 126, 2991-3012.

Mitchell, H. L., and P. L. Houtekamer, 2000: An adaptive ensemble Kalman filter. Mon. Wea. Rev., 128, 416-433.

,-- , and G. Péllerin, 2002: Ensemble size, balance, and model-error representation in an ensemble Kalman filter. Mon. Wea. Rev., 130, 2791-2808.

Molteni, F., R. Buizza, T. N. Palmer, and T. Petroliagis, 1996: The ECMWF ensemble prediction system: Methodology and validation. Quart. J. Roy. Meteor. Soc., 122, 73-119.

Morss, R. E., 1998: Adaptive observations: Idealized sampling strategies for improving numerical weather prediction. Ph.D. dissertation, Massachusetts Institute of Technology, 225 pp. [Available from UMI Dissertation Services, P.O. Box 1346, 300 N. Zeeb Rd., Ann Arbor, MI 48106-1346.]

—_, K. Emanuel, and C. Snyder, 2001: Idealized adaptive observation strategies for improving numerical weather prediction. $J$. Atmos. Sci., 58, 210-234.

Mureau, R., F. Molteni, and T. N. Palmer, 1993: Ensemble prediction 
using dynamically conditioned perturbations. Quart. J. Roy. Meteor. Soc., 119, 299-323.

Parrish, D. F., and J. C. Derber, 1992: The National Meteorological Center's spectral statistical-interpolation analysis system. Mon. Wea. Rev., 120, 1747-1763.

Pham, D. T., 2001: Stochastic methods for sequential data assimilation in strongly nonlinear systems. Mon. Wea. Rev., 129, 1194-1207.

Preisendorfer, R. W., 1988: Principal Component Analysis in Meteorology and Oceanography. Elsevier Press, $425 \mathrm{pp}$.

Press, W. H., S. A. Teukolsky, W. T. Vetterling, and B. P. Flannery, 1992: Numerical Recipes in Fortran. 2d Ed. Cambridge University Press, $963 \mathrm{pp}$.

Rabier, F., A. McNally, E. Andersson, P. Courtier, P. Undén, J. Eyre, A. Hollingsworth, and F. Bouttier, 1998: The ECMWF implementation of three-dimensional variational assimilation (3DVar). II: Structure functions. Quart. J. Roy. Meteor. Soc., 124, 18091829.

Smolarkiewicz, P. K., and L. G. Margolin, 1994: Variational solver for elliptic problems in atmospheric flows. Appl. Math Comput. Sci., 4, 527-551.

Snyder, C., and T. M. Hamill, 2001: Lyapunov stability of a turbulent baroclinic jet in a quasigeostrophic model. J. Atmos. Sci., submitted.
,-- , and S. Trier, 2002: Linear evolution of error covariances in a quasigeostrophic model. Mon. Wea. Rev., in press.

Thépaut, J.-N., R. N. Hoffman, and P. Courtier, 1993a: Interactions of dynamics and observations in a four-dimensional variational assimilation. Mon. Wea. Rev., 121, 3393-3414.

_ D. Vasiljevic, P. Courtier, and J. Pailleux, 1993b: Variational assimilation of conventional meteorological observations with a multilevel primitive equation model. Quart. J. Roy. Meteor. Soc., 119, 153-186.

Tippett, M. K., S. E. Cohn, R. Todling, and D. Marchesin, 2000: Low-dimensional representation of error covariance. Tellus, 52A, 533-553.

Toth, Z., and E. Kalnay, 1993: Ensemble forecasting at NMC: The generation of perturbations. Bull. Amer. Meteor. Soc., 74, 2317 2330.

_ , and —_, 1997: Ensemble forecasting at NCEP and the breeding method. Mon. Wea. Rev., 125, 3297-3319.

van Leeuwen, P. J., 1999: Comments on "Data assimilation using an ensemble Kalman filter technique." Mon. Wea. Rev., 127, 13741377.

Whitaker, J. S., and T. M. Hamill, 2002: Ensemble data assimilation without perturbed observations. Mon. Wea. Rev., 130, 19131924.

Wilks, D. S., 1995: Statistical Methods in the Atmospheric Sciences: An Introduction. Academic Press, 467 pp. 